\title{
Analysis of Conditioned Courtship in dusky-Andante Rhythm Mutants of Drosophila
}

\author{
Bruno van Swinderen ${ }^{1}$ and Jeffrey C. Hall ${ }^{2}$ \\ Department of Biology \\ Brandeis University \\ Waltham, Massachusetts 02254
}

\section{Abstract}

Genetic connections between learning and rhythmicity were suggested to have been established in a previous study, in part because the dusky $y^{\text {Andante }}\left(d y^{\text {And }}\right)$ mutation in Drosopbila disrupted both behaviors. $d y^{A n d}$, isolated as a slow-clock variant, was reported to cause an approximately fourfold decrement in courtship-suppression conditioning. These effects have been reexamined; the experiments were buttressed by testing the effects of several recently isolated mutations at the dusky locus, along with the original And allele that had been induced there. The reexamination was also prompted by anatomical concerns, certain of which have recently focused on $\boldsymbol{d y}$-induced decrements in cell size, but only in terms of wing morphology. Another anatomical issue involves the discovery of a neuronal pathway that seems to connect circadian pacemaker cells to a structure in the Drosopbila brain that is involved in learning. In observer-blind experiments, however, it was found that neither pacemaker-slowing (Andante-like) $d y$ mutations nor others that cause no rhythm defects produced subnormal conditioned courtship. Moreover, in the adult brain of a slow-clock $d y^{A n d}$ mutant, no axonal pathway defects were readily discernible and putative pacemaker neurons appeared to be normal in size.

\footnotetext{
${ }^{1}$ Present address: Department of Microbiology, Washington University Medical School, St. Louis, Missouri 63110. ${ }^{2}$ Corresponding author.
}

\section{Introduction}

Courtship-suppression conditioning in Drosophila has been used to test a variety of genetic variations beyond those involved in reproductive behavior alone. For example, the classic learning and memory mutants yielded diminished aftereffects of a male's previous experiences with reproductively unreceptive flies (for reviews, see Siegel et al. 1984; Hall 1994a; Greenspan 1995). Consequently, this behavioral assay has proved useful for asking whether other kinds of mutants, strains, and molecularly manipulated transgenics would exhibit learning defects (Heisenberg 1980; Collins and Hewitt 1981; Cowan and Siegel 1984; Tempel et al. 1984; Zawistowski and Richmond 1985, 1988; Zawistowski 1988; Gailey and Siegel 1989; Griffith et al. 1993, 1994; Bouhouche and Choulli 1994; O'Dell 1994).

Perhaps the most surprising effects on courtship suppression were those reported for rhythm mutations, such as period, Andante (And), and "phase angle" ( $p s i)$. These X-chromosomal and autosomal mutants, isolated on the basis of rhythm abnormalities (and all exhibiting longer-than-normal periodicities), were found to be defective in courtship suppression (Jackson et al. 1983). Such decrements were at least as great as those caused by dunce, rutabaga, or amnesiac mutations (cf. Gailey et al. 1982, 1984). It was as if a clock that runs slowly would not allow information to be stored or retrieved in an optimal manner. More generally, the possibility that rhythm-related mechanisms might subserve animal learning has long been entertained (e.g., Jenkins and Dallenbach 1924; Smith 1985; Stone et al. 1989).

The most salient rhythm variants in Drosophila are the period (per) mutants, which include strains whose circadian-clock periods are several hours longer than normal (for review, see

LEARNING \& MEMORY 2:49-61 ๔ 1995 by Cold Spring Harbor Laboratory Press ISSN1072-0502/95 \$5.00

$$
\begin{array}{llllllllllllllll}
\hline & E & A & R & N & I & N & G & \begin{array}{l}
\boldsymbol{Z} \\
49
\end{array} & M & E & M & O & R & Y
\end{array}
$$


Konopka 1987). Males expressing per-long (per ${ }^{L}$ ) were among those found to exhibit mediocre conditioned courtship suppression (Jackson et al. 1983). Yet, such learning defects for the per ${ }^{L}$ mutants were found not to be extendible in two ways: First, per transgenics with $\sim 27-h r$ periods (vs. the normal value of $24 \mathrm{hr}$ ) exhibited normal courtship conditioning. Second, neither per $^{L}$ nor other kinds of per mutants (short-period or arrhythmic) showed abnormal learning or memory scores in a Pavlovian olfactory learning task (Gailey et al. 1991). The only per-related finding from the earlier study (Jackson et al. 1983) that was reproducible involved a per $^{L 1}$-mediated decrement in male learning, as is usually effected by his courtship of fertilized females (Gailey et al. 1991). Extended conditioning periods, however, eliminated any difference between per $^{L 1}$ and wild-type controls. Learning in the per ${ }^{L 1}$ mutant also was normal when tested with immature males; furthermore, the per $^{\mathrm{L}}$ mutant's learning in both the fertilizedfemale and immature-male assays was normal (Gailey et al. 1991). The one instance of a per ${ }^{2}$-associated learning abnormality appeared to result from a genetic-background effect, primarily because the intragenic site change (cf. Baylies et al. 1987; Huang et al. 1993) in the per ${ }^{L 1}$ mutant was found to be identical to that of per $^{L 2}$ (Gailey et al. 1991).

In the earlier experiments involving rhythm defects and learning, the most dramatically defective mutant was And, notwithstanding the fact that this mutant's circadian clock runs only 1-2 hr more slowly than normal (Konopka et al. 1991; Newby et al. 1991). A set of novel And-like mutants was induced by mutagenesis, and the isolation of these variants was accompanied by analysis of their pleiotropic effects (Newby et al. 1991). In this regard, it was definitively established that And-like mutants occur at the dusky (dy) locus (Newby et al. 1991; cf. Konopka et al. 1991). Yet, only some of the new mutants, with their $d y$-wing defects, had longer-than-normal rhythm periods. It was suggested, nevertheless, that the "small wingcell" phenotype (which is part of $d y$ 's pleiotropy) might extend to brain cells underlying the fly's rhythms. Presumably, a smaller-than-normal volume for such "pacemaker neurons" would cause the clock to run at a relatively slow pace (Newby et al. 1991).

The existence of the new $d y$ mutants-both the normal and slow-rhythm types-is one reason that prompted an attempt to replicate and extend the original $d y^{A n d}$ learning defects. Moreover, sub- sequent to the earliest learning experiments on And itself, Drosopbila's circadian pacemaker cells have been identified as a result of genetic-mosaic and per-transgenic experiments (Ewer et al. 1992; Frisch et al. 1994). In addition, axonal projections emanating from a subset of the per-expressing neurons send processes into the CNS proper, where they terminate near the calyces of the mushroom bodies (Helfrich-Förster and Homberg 1993; Helfrich-Förster 1995). Those structures are involved in the control of (olfactory) associative learning (for review, see Davis 1993; also see de Belle and Heisenberg 1994). These recent anatomical observations suggest anew that a "clock mutant could be a learning mutant". $d y^{\text {And }}$ flies and their allelic relatives might have brain-structural abnormalities after all (in addition to exhibiting their externally visible wing defects). Thus, according to an inference reasonably drawn from the results of Helfrich-Förster and Homberg (1993), $d y^{A n d}$-related brain anomalies could at once produce a defective biological clock and abnormal learning machinery. Extensive behavioral and neuroanatomical analyses of the original And mutation and the newly isolated $d y$ mutants have, however, shown this hypothesis to be invalid.

\section{Materials and Methods}

\section{DROSOPHILA STRAINS AND CULTURE CONDITIONS}

Flies were raised on cornmeal, sugar, and yeast medium. Most $d y$-mutant strains were obtained from F.R. Jackson (Worcester Foundation for Experimental Biology, Shrewsbury, MA). They had been induced by either chemical or gammaray mutagenesis (Newby et al. 1991). The ionizing-radiation treatments, followed by screening for novel $\boldsymbol{d y}$-wing variants, yielded four new mutant alleles: $d y^{n 1}, d y^{n 2}, d y^{n 3}$, and $d y^{n 4}$. Similarly, the ethylmethane sulfonate treatments led to the $d y^{r 2}$, $d y^{r 3}, d y^{r 4}$, and $d y^{r 5}$ alleles. One $d y$ mutant reported by Newby et al. (1991), $d y^{n 2}$, was not investigated in the present behavioral experiments owing to its near-normal appearance (see description of "blind-testing" procedure, below). The original $d y$-related rhythm variant, $d y^{\text {And }}$, had been maintained here for many years (having been obtained from R.J. Konopka, a long time before it was reported; Konopka et al. 1991). It, along with the first eight $d y$ 's listed above, is identifiable by wings that are smaller and darker than in wild-type

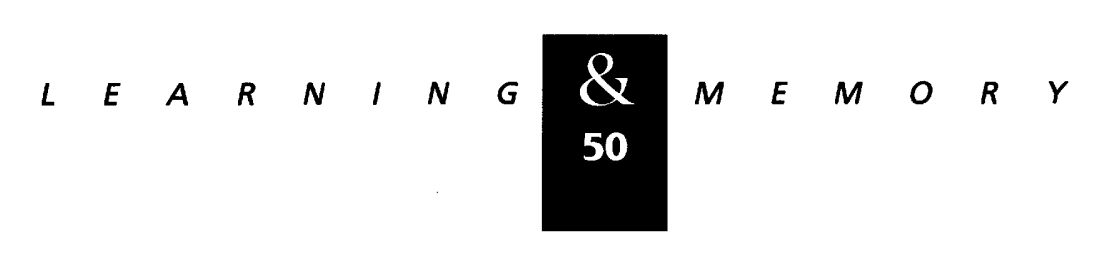


adults. This darkening is not a pigmentation problem but is caused by the fact that individual wing cells of $d y$ flies are smaller than normal (see Newby et al. 1991 and references therein).

The eight recently isolated $d y$ mutants were maintained (in true-breeding stocks) whose females are attached-X, $y f$ females. $d y$ males from each such line were outcrossed to Canton-S wild type for two "recombination cycles". The resulting recombinant lines, extracted by simply selecting for $d y$-wing males and (homozygous) females, were the ones used in all behavioral tests (see below).

For certain of the histochemical experiments (see below), the $d y^{\text {And }}$ mutation was introduced into stocks that also carried a fusion gene involv. ing the 5' half of Drosopbila's period locus, ligated in-frame to lacZ from Escherichia coli (Liu et al. 1988, 1991); this transgene is located on chromosome 2 and is homozygous viable.

\section{BEHAVIORAL PROCEDURES}

\section{CIRCADIAN RHYTHMS}

Adult males were collected soon after eclosion and entrained for 3 days to a 12:12 light-dark (LD) cycle in a constant-condition room $\left(25^{\circ} \mathrm{C}\right.$, $75 \%$ humidity). Flies were then individually placed in a circadian rhythm monitoring device (see Hamblen et al. 1986, for details), in constant darkness (DD); each fly's activity was recorded automatically for 2 weeks. Statistical analysis of the locomotor activity events was done on a VAX/VMS computer system (cf. Hamblen-Coyle et al. 1992; Wheeler et al. 1993).

\section{COURTSHIP}

The courtship-suppression paradigm, involving male behavior as affected by interactions with fertilized females, was used to determine whether there are different learning abilities among the $d y$ lines. In all cases, a given strain was coded as to the $d y$ allele it carried, to militate again observer bias. The "blind controls" could not be wild-type (hence normal-winged) animals and were, instead, deemed to be $d y$ mutants that do not have an altered circadian-clock pace (see Introduction and below; Table 1), with the "experimentals" being
Table 1: Circadian rhythms of dusky mutants

\begin{tabular}{lc}
\hline Genotype & Circadian $\tau(\mathrm{h} \pm$ S.E.M.) \\
\hline$d y^{\text {And }}$ & $26.2 \pm 0.3$ \\
$d y^{n 1}$ & $26.0 \pm 0.4$ \\
$d y^{n 3}$ & $25.5 \pm 0.3$ \\
$d y^{n 4}$ & $26.0 \pm 0.4$ \\
$d y^{r 2}$ & $24.3 \pm 0.3$ \\
$d y^{r 3}$ & $24.4 \pm 0.2$ \\
$d y^{r 4}$ & $24.2 \pm 0.3$ \\
$d y^{r 5}$ & $24.4 \pm 0.4$ \\
Wild type & $24.1 \pm 0.3$ \\
\hline
\end{tabular}

Free-running circadian periodicities, resulting from monitorings of adult locomotor activity, were determined as described in Materials and Methods, which include reference to the $\chi^{2}$ periodogram analyses used to provide best estimates of these period $(\tau)$ values. Eight to 10 individuals (all of whom behaved in a significantly periodic manner) were tested for each of these nine genotypes; the final one listed refers to males from a Canton-S strain.

dy mutants that exhibit longer-than-normal rhythm periods as well as aberrant wing morphology.

During certain (rather wide) windows of time in 12:12 LD schedules, 5-day-old test males were paired and "conditioned" with 5-day-old fertilized Canton-S (wild-type) females. Almost all such tests were performed within $5 \mathrm{hr}$ after lights-on and within $2 \mathrm{hr}$ before lights-off; these windows are near the times of general locomotion-maxima for flies of this species (e.g., Hamblen-Coyle et al. 1992; Wheeler et al. 1993). Jackson et al. (1983), however, has indicated that courtship-suppression scores seemed not to be affected by the timesthroughout the daylight hours-when their tests were performed.

Male-female pairings (in the current experiments) were effected for 30-min training (or control) periods, in cleaned porcelain observation wells covered by glass slides (cf. Gailey et al. 1986, 1991). The fertilized females used in these tests had each been observed to mate with one Canton-S male, 18-24 hr previously. In the control paradigm, 5-day-old males were placed alone in the observation wells for $30 \mathrm{~min}$ (cf. Jackson et al. 1983, whose controls involved pairing a previously naive male with an immature virgin female for $30 \mathrm{~min}$ ).

For testing, conditioned or control males were gently aspirated into new individual obser-

$$
\text { ……‥ }
$$


vation wells (immediately after the 30 min of pairing or solitary confinement). There-within 1-2 min-they were presented with a 5-day-old etherized, Canton-S virgin female. A courtship index (CI) was then measured for each male by accumulating (using electronic timers) the proportion of the observation periods during which the test male directed any courtship actions toward the ether-immobilized female (see reviews by Tompkins 1984, 1989; Hall 1994a, for further details about $\mathrm{CI}$ determinations). An observation period was terminated as soon as the etherized female regained mobility, which was usually in the range of 6-15 min.

CI values were processed by angular (arcsine) transformations (Sokal and Rohlf 1981, p. 427), to correct for the abnormal distribution of residuals in such raw data. This unevenness may be caused by "floor" and "ceiling" effects, whereby data points at the high and low extremes exert disproportionate leverage on the regression. This minidiscussion is provided, against a background of floor and ceiling effects (mainly the former) not having been corrected for in previous courtship. conditioning experiments-notably those involving rhythm mutants (Jackson et al. 1983).

CI values were further processed by two different methods: (1) One way to express the extent to which males of a given genotype learned is simply to consider the difference between the $\mathrm{CI}_{\text {cont }}$ and $\mathrm{CI}_{\text {exp }}$ values (though this metric is subject to the aforementioned floor effects, i.e., when $\mathrm{CI}_{\text {cont }}$ values are relatively low); the standard errors of the mean associated with such differences (Table 2) were estimated as in Sokal and Rohlf (1981, p. 226). (2) The courtship learning index $\left(\lambda_{c}\right)$ was computed for each individual mutant:

$$
\lambda_{\mathrm{C}}=\left[1-\left(\mathrm{CI}_{\mathrm{exp}} / \mathrm{CI}_{\text {cont }}\right)\right] 100
$$

as originally devised by Gailey et al. (1982, 1984). Because the estimate of the $\mathrm{CI}_{\exp } / \mathrm{CI}_{\text {cont }}$ ratio is biased (cf. Grossman and Norton 1980), it was corrected by $\left(\mathrm{CI}_{\text {exp }} / \mathrm{CI}_{\text {cont }}\right) \times\left[1+\operatorname{Var} \mathrm{CI}_{\text {cont }} /\left(\mathrm{CI}_{\text {cont }}\right)^{2}\right]$. After subtracting the corrected ratio from 1.0, the resulting $\lambda_{c}$ has a value of $O$ in a case for which the behaviors of experimental and control

Table 2: Courtship suppression assays of long-period ( $(L)$ and circadian-normal $(N)$ dusky mutants

\begin{tabular}{|c|c|c|c|c|c|c|}
\hline \multirow[b]{2}{*}{ Mutants } & \multicolumn{2}{|c|}{ Raw } & \multicolumn{2}{|c|}{ Transformed } & \multirow[b]{2}{*}{$\mathrm{Cl}_{\text {cont }}-\mathrm{Cl}_{\text {exp }}$} & \multirow[b]{2}{*}{$\lambda_{\mathrm{C}}$} \\
\hline & $\mathrm{Cl}_{\text {cont }}$ & $\mathrm{Cl}_{\exp }$ & $\mathrm{Cl}_{\text {cont }}$ & $\mathrm{Cl}_{\exp }$ & & \\
\hline$d y^{A n d}(\mathrm{~L})$ & $43 \pm 8$ & $12 \pm 5$ & $37 \pm 6$ & $14 \pm 4$ & $23 \pm 7$ & $61 \pm 12$ \\
\hline$d y^{n 1}(L)$ & $47 \pm 8$ & $15 \pm 4$ & $41 \pm 6$ & $18 \pm 3$ & $23 \pm 7$ & $55 \pm 10$ \\
\hline$d y^{n 3}(\mathrm{~L})$ & $22 \pm 7$ & $5 \pm 3$ & $20 \pm 5^{*}$ & $6 \pm 3^{*}$ & $14 \pm 6$ & $68 \pm 17$ \\
\hline$d y^{n 4}(L)$ & $24 \pm 6$ & $5 \pm 4$ & $23 \pm 5^{*}$ & $7 \pm 3^{*}$ & $16 \pm 6$ & $68 \pm 15$ \\
\hline$d y^{r 2}(N)$ & $48 \pm 9$ & $20 \pm 7$ & $41 \pm 7$ & $19 \pm 6$ & $22 \pm 9$ & $52 \pm 17$ \\
\hline$d y^{r 3}(N)$ & $50 \pm 8$ & $26 \pm 7$ & $43 \pm 6$ & $24 \pm 6$ & $19 \pm 8$ & $43 \pm 16$ \\
\hline$d y^{r 4}(N)$ & $48 \pm 8$ & $26 \pm 6$ & $40 \pm 6$ & $23 \pm 5$ & $17 \pm 8$ & $41 \pm 15$ \\
\hline$d y^{r 5}(N)$ & $50 \pm 9$ & $23 \pm 7$ & $42 \pm 7$ & $22 \pm 6$ & $20 \pm 9$ & $46 \pm 17$ \\
\hline Wild type $(\mathrm{N})$ & $67 \pm 6$ & $29 \pm 8$ & $56 \pm 4$ & $28 \pm 6$ & $28 \pm 7$ & $50 \pm 11$ \\
\hline
\end{tabular}

Courtship indices $(\mathrm{Cl} \pm$ S.E.M.) for control (cont) and experimental (exp) tests of eight independently isolated dy mutants and wild-type (Canton-S) flies were determined as described in Materials and Methods; $n=20$ for each of the values in the first four data columns. (L) A long-period mutant; $(\mathrm{N})$ a strain that exhibits normal circadian $\tau^{\prime} \mathrm{s}$ (cf. Table 1). Raw $\mathrm{Cl}$ values ( \pm S.E.M.) are given in the first two data columns; arcsine-transformed versions of such values (see Materials and Methods) are in the third and fourth columns. Using transformed $\mathrm{Cl}^{\prime}$, control vs. experimental values (whose differences appear in the fifth column) were compared statistically; all were significantly different: $P$ values for the nine intragenotype comparisons ranged from 0.02 to $4 \times 10^{-4}$. Courtship learning indices $\left(\lambda_{C}\right)$ were calculated (using the transformed Cl cont and $\mathrm{Cl}_{\text {exp }}$ values), as described in the text (also see Gailey et al. 1982, 1984). Pairwise, intergenotype comparisons of these $\lambda_{C}{ }^{\prime}$ s indicated no significant differences ( $P$ values were 0.21 or higher). $d y^{n 3}$ and $d y^{n 4}$ exhibited significantly low courtship vigor in general [by ANOVA; see asterisks $\left({ }^{*}\right)$; cf. Materials and Methods] and lower-than-normal locomotor-activity levels (see Table 1); this made the $\mathrm{Cl}_{\text {cont }}-\mathrm{Cl}_{\text {exp }}$ values relatively low for these two dy mutants. Yet, intergenotype pairwise comparisons among all the $\mathrm{Cl}$-difference values indicated none was significant: $P$ values were 0.07 or higher, and-owing to the large number of pairwise comparisons made $-\alpha$ had to be corrected to a value much lower than the benchmark one of 0.05 , i.e., by the function $1-(.95)^{1 / k}$ (where $k$ is the number of such comparisons; Sokal and Rohlf 1981, p. 241).

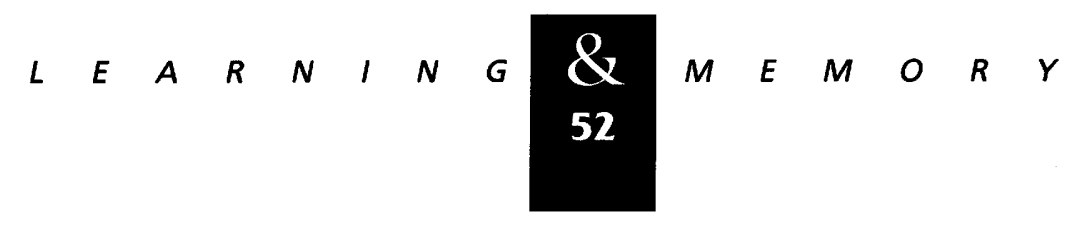


males of a given genotype, tested in parallel, are alike within a line; thus, "no learning" is inferred to have occurred; "perfect learning" (100) is computed in a case where courtship-suppression, as a result of training in the presence of the fertilized female, is absolute $\left(\mathrm{CI}_{\exp }=0\right)$, with appreciable $\mathrm{CI}_{\text {cont }}$ values being obtained (as is always the case). The standard errors associated with the learning indices could not be computed directly (owing to the subtraction of one group mean from another: see above) and so were estimated by the function: (Mean $\left.\mathrm{CI}_{\text {exp }}\right)^{2} /\left(\text { Mean } \mathrm{CI}_{\text {cont }}\right)^{2} \times[\mathrm{Var}$ $\left.\mathrm{CI}_{\text {exp }} /\left(\text { Mean } \mathrm{CI}_{\text {exp }}\right)^{2}+\operatorname{Var} \mathrm{CI}_{\text {cont }} /\left(\text { Mean } \mathrm{CI}_{\text {cont }}\right)^{2}\right]$ (cf. Tully and Hirsch 1982).

CI values (of the transformed variety) were compared among genotypes, using a two-way analysis of variance; the "main effects" considered in these ANOVAs were genotype and paradigm (control or experimental); a significant F-ratio determination was followed by "planned" comparisons between genotypes. Owing to the fact that there is no series of individual $\lambda_{C}$ or $\mathrm{CI}_{\text {cont }}{ }^{-\mathrm{CI}_{\exp }}$ values for a given genotype, pairwise comparisons between learning indices and the control versus experimental CI differences were performed by $\boldsymbol{t}$-tests (Sokal and Rohlf 1981). Intragenotype differences between $\mathrm{CI}_{\text {cont }} \mathrm{s}$ and $\mathrm{CI}_{\text {exp }}$ s were also compared by $t$-tests.

\section{HISTOCHEMISTRY}

Stainings of per gene-expressing cells in the CNS and PNS of adult heads was performed by cryostat sectioning ( $10 \mu \mathrm{m}$, horizontal plane), followed by (1) application- to sections of flies from the $d y^{A n d}$-carrying, per-lac $Z$ transgenic strain (see above) - of an $\alpha-\beta G A L$ antibody (see Liu et al. 1991 for procedural details) [specifically, we found that an $\alpha$ - $\beta$ GAL from mouse (Promega) gave sharper staining of "per neurons" than did other commercially available antibodies made against this bacterial enzyme (e.g., as made in rabbits, purchased from Cappel)] and (2) use of an anti-ARRESTIN-2 antibody ( $\alpha$-ARR2) (cf. Dolph et al. 1993), which happens to mark all photoreceptor cells and CNS neurons that express per (B. Frisch, J. Wagner, B. van Swinderen, M. Kaneko, and J.C. Hall, in prep.). This antibody was a gift of $P$. Dolph and C.S. Zuker (University of California, San Diego, La Jolla). The $\alpha$-ARR2 reagent had been made in rabbit against a synthetic peptide (P. Dolph and C.S. Zuker, pers. comm.). We applied this antibody to frozen sections and stained them as in Siwicki et al. (1988). Some of the sections were prepared from fly heads that had been fixed overnight in paraformaldehyde; this yielded better labeling of neuronal projections (see below). Dolph et al. (1993) and B. Frisch, J. Wagner, B. van Swinderen, M. Kaneko, and J.C. Hall (in prep.) have shown that the arr2 gene's normal, bona fide product is expressed in photoreceptors of the compound eye and ocelli and in a putative extraocular photoreceptive structure (cf. Hofbauer and Buchner 1989). $\alpha$-ARR2 also leads to staining of all or nearly all per neurons and many of the projections from them (B. Frisch, J. Wagner, B. van Swinderen, M. Kaneko, and J.C. Hall, in prep.; see Table 3 footnote). That the perikarya and neurites are per-expressing neuronal entities in the adult brain is inferred not only from the similar cellular positions and qualities that are observed when applying $\alpha$-PER or $\alpha$-ARR2 in separate studies, but also as a result of performing double-label experiments (B. Frisch, J. Wagner, B. van Swinderen, M. Kaneko, and J.C. Hall, in prep.). However, the latter antigen seems to be cross-reacting material owing to the continued presence of CNS signals in an arr2-null mutant (B. Frisch, J. Wagner, B. van Swinderen, M. Kaneko, and J.C. Hall, in prep.), which had been isolated by Dolph et al. (1993).

\section{Results}

\section{CONDITIONED BEHAVIOR OF $d y$ MUTANT MALES}

Certain $d y$ mutations were reported to produce relatively long-period rhythms ( $>25 \mathrm{hr}$ ), whereas others were ostensibly normal (Konopka et al. 1991; Newby et al. 1991). Retesting outcrossed versions of these particular mutants yielded circadian-rhythm periodicities (exemplified in Fig. 1) similar to those reported previously (cf. Newby et al. 1991). Thus, four lines $\left(d y^{A n d}\right.$, $\left.d y^{n 1}, d y^{n 3}, d y^{n 4}\right)$ continue to display longer-thannormal periods, and four others $\left(d y^{r 2}, d y^{r 3}, d y^{r 4}\right.$, $d y^{r 5}$ ) still give normally rhythmic behavior (Table 1). Within the slow-clock category, however, certain variations were noted: $d y^{n 3}$ and $d y^{n 4}$ seemed less active than $d y^{n 1}$ or $d y^{A n d}$ (exemplified in Fig. 1 ), although the $\sim 25$ - to $26-\mathrm{hr}$ rhythmicities computed for all four of these types were robust (in terms of analytical determination of the relevant " $\tau$ " values; Table 1 ).

$d y^{A n d}$, née $A n d$, is the one long-period $d y$ mu-

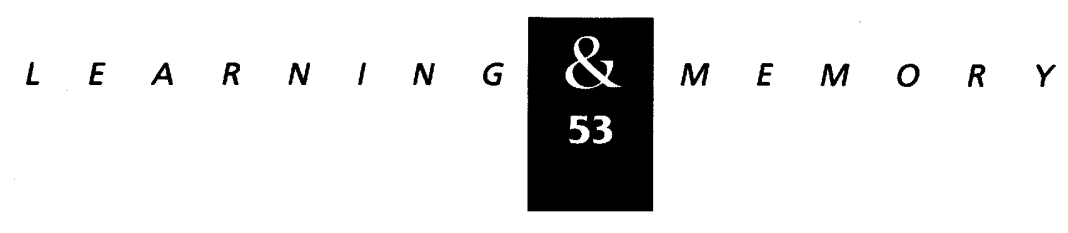


van Swinderen and Hall

Table 3: $\alpha$-ARR2 immunohistochemistry of $\mathrm{dy}^{\text {And }}$ mutant adult brains

\begin{tabular}{lcccc}
\hline & Present? & $\begin{array}{c}\text { Diameter } \\
(\mu \mathrm{m})\end{array}$ & Number & $\begin{array}{c}\text { Vertical dispersal } \\
(\mu \mathrm{m})\end{array}$ \\
\hline Dorsal posterior cells & Yes* $^{*}$ & $4-8$ & $1-2$ & 20 \\
Dorsal-lateral cells & Yes* & $4-6$ & $1-2$ & 10 \\
Ventral-lateral cells & Yes & $10-12$ & $4-6$ & $\sim 35$ \\
Central posterior cells & Yes & $2-8$ & $4-6$ & 30 \\
H-B (photoreceptor?) cells & Yes & - & - & - \\
Ventrolateral $\rightarrow$ central connections & Yes & - & - & - \\
Dorsal loop & Yes & - & - & - \\
\hline
\end{tabular}

Five $d y^{\text {And }}$ males were stained with $\alpha$-ARR2 using the same protocol as applied to wild-type controls (see below). The stained structures listed in the first three rows of the left-most column correspond to the per-expressing dorsal neurons (DNs) and lateral neurons (LNs) of Siwicki et al. (1988), Zerr et al. (1990); and Fisch et al. (1994); the Hofbauer-Buchner cells are putative extraocular photoreceptors (Hofbauer and Buchner 1989), which express per, as do all other external photoreceptors (Siwicki et al. 1988; Ewer et al. 1992). The various cell types were observed in all five mutant individuals prepared for these histochemical observations, except for the two types denoted by asterisks $\left({ }^{*}\right)$; these were not seen in one fly (further information on these seven anatomical features is given in Materials and Methods). The ranges implied by dashes are the extreme interanimal values for these anatomical measurements. Values in the right-most column represent the roughly averaged extents—in the dorsal-to-ventral direction-over which these four groups of cells were distributed. The ventral cluster of lateral neurons sends an apparent projection to the region of cells stained in a relatively "central posterior" region: from the region of the latter, a "loop" of stained axon bundles projects in a dorsal direction, terminating in a neuropil region near that of the mushroom bodies. The several elements of these cell-body and neurite stainings were first established in sections of nine wild-type heads (methodological and informational details to be reported elsewhere; B. Frisch, J. Wagner, B. van Swinderen, M. Kaneko, and J. C. Hall, in prep.). As implied by the tabulated entries and the foregoing statements in this legend, the staining patterns and associated numerical features (see the three data columns above) were essentially identical between these normal preparations and those of the $d y^{\text {And }}$ mutant heads.

tant for which learning has been assayed (Jackson et al. 1983). The courtship learning index in a previous test of wild-type males (circadian $\tau$ for that strain, $23.8 \mathrm{hr}$ )-involving mated females and untransformed CI values-was 91 , with the parallel test of one of the above mutants, $d y^{\text {And }}[\tau$ (then) $=25.5 \mathrm{hr}$, yielding a $\lambda_{\mathrm{C}}$ of 23 (Jackson et al. 1983). Note that the current control $\lambda_{C}$ value (50) is considerably lower than that of Jackson et al. (1983). This is not uncommon in courtship suppression tests (see Hall 1994a for the pertinent literature), performed with different strains (irrespective of mutants) and at widely separate times. The key comparisons would involve courtship suppression scores determined contemporaneously from testing flies with similar genetic backgrounds (see Materials and Methods).

Using this kind of genetic design and in light of the problematical nature of $p^{L}{ }^{L}$-associated learning phenotypes (Gailey et al. 1991), we reexamined and extended the original experiments on $d y$ 's learning abilities. The extension included an internal control: Courtship-suppression phenotypes of the long-period and clock-normal $d y$ mutants were compared in an experimenter-blind manner (possible, because all of these lines have the $d y$-wing phenotype).

Table 2 summarizes results from the current courtship experiments. First, all differences in arcsine-transformed $\mathrm{CI}$ values from control $\left(\mathrm{CI}_{\text {cont }}\right)$ versus experimental $\left(\mathrm{CI}_{\text {exp }}\right.$, i.e., postmated female) groups within a genotype were significant (see Table 2 footnote). Second, transformed $\mathrm{CI}_{\text {exp }}$ values for most of these $d y$ mutants were not significantly different from the wild-type value nor from one another. The exceptions involved $d y^{n 3}$ and $d y^{n 4}$ flies, for which both $\mathrm{CI}_{\text {exp }} \mathrm{s}$ and $\mathrm{CI}_{\text {cont }} \mathrm{s}$ were significantly lower than the corresponding values for the other mutant or wild-type strains (Table 2, see asterisks)-notwithstanding the fact that these and all of these $d y$ alleles had been outcrossed to wild type and had true-breeding mutant animals reextracted before commencing the behavioral experiments (see Materials and Methods). The relatively nonvigorous courtship performances of the $d y^{n 3}$ and $d y^{n 4}$ mutants produced a floor effect when calculating the absolute differences between the $\mathrm{CI}_{\text {cont }}$ and $\mathrm{CI}_{\text {exp }}$ values (Table 2) for these two $d y$ mutants. This difference is another way to reflect the extent to which a given

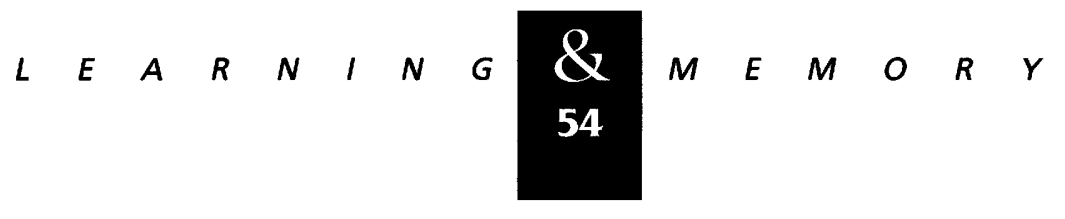




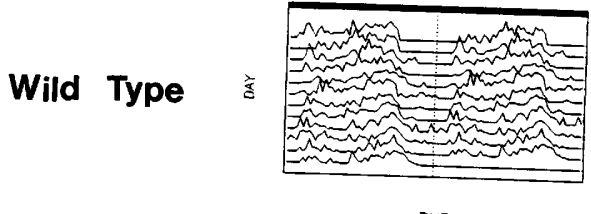

TIME

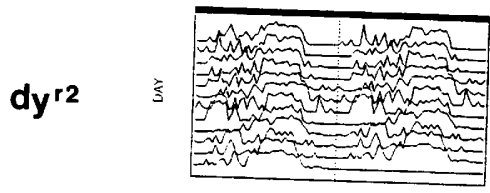

TIME

dy ${ }^{\text {3 }}$

咅

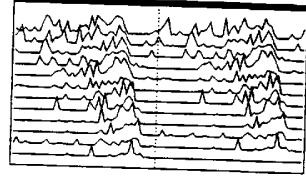

TME

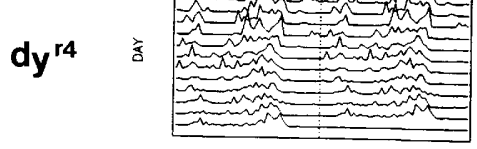

TIME

dy $y^{\text {5 }}$

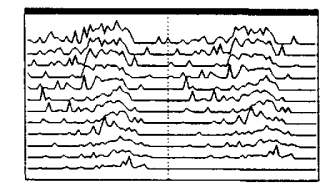

TIME dy And

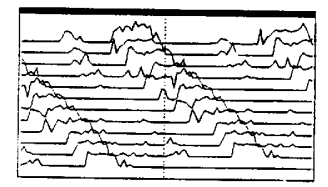

TIME

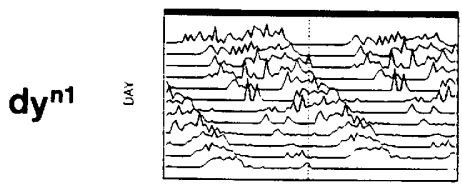

TME

dyn $^{\text {3 }}$

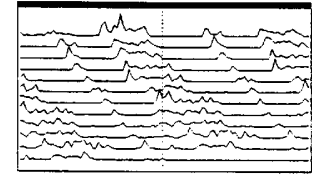

TIME

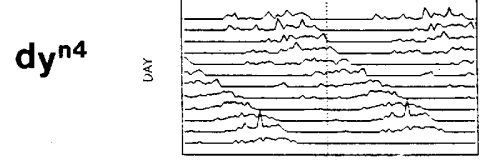

TIME
Figure 1: Actograms displaying normal and mutant circadian rhythms of locomotor activity. Each plot shows the activity of one male fly, recorded in DD over a period of $\sim 2$ weeks. "TIME" axes represent two consecutive $24-\mathrm{hr}$ blocks, such that the horizontal "darkness" bars at the top of each "double plot" represent $48 \mathrm{hr}$. Double plotting means that days 1-2 of DD behavior are displayed on the top line of a given actogram, days 2-3 on the next line, etc. The rough peaks of activity-event values occurred vertically with respect to one another, in the wild-type record and those of the four $d y$ mutants at left, which in general have normally paced clocks (Table 1 ). In the four right-hand plots, the major peaks occur appreciably later on consecutive days, typifying the $>25-\mathrm{hr}$ rhythm periods that are caused by these four dy alleles (Table 1). male learned from his experience with a fertilized female (see Materials and Methods and below). Nevertheless, none of the mutant $\mathrm{CI}_{\text {cont }}-\mathrm{CI}_{\text {exp }}$ difference values were different from one another (see Table 2 footenote). The latter $\mathrm{CI}_{\text {cont }}-\mathrm{CI}_{\exp }$ value for wild type was 28-nominally (yet not significantly) higher than the (interstrain) averages for the long-period and rhythm-normal $d y$ mutants; these were very similar to each other, $19(n=4$ strains) and $20(n=4)$, respectively (Table 2$)$.

The courtship-learning index $\left(\lambda_{\mathrm{C}}\right)$ was developed in part to reflect relative, as opposed to absolute, differences between $\mathrm{CI}_{\text {cont }}$ and $\mathrm{CI}_{\exp }$ values. Here again, mutant $\lambda_{C} s$ did not differ significantly from each other or from those of the wild type (see Table 2 footnote). Moreover, ranking the nominally highest to lowest $\lambda_{\mathrm{C}}$ (e.g., $d y^{n 3}, d y^{n 4}$ versus $\left.d y^{r 3}, d y^{r 4}\right)$ revealed no correlation with these mutants' period values (cf. Table 1 ). In fact, the interstrain $\lambda_{C}$ averages for the normally periodic mutants (46) was nominally worse than the overall index (63) for the slow-clock lines. In this regard, the $\mathrm{CI}_{\text {cont }}-\mathrm{CI}_{\text {exp }}$ difference values (and the statistic newly applied for comparing them, Table 2) may better reflect the rather uniform courtshiplearning abilities exhibited by these two categories of mutants.

\section{BRAIN ANATOMY OF A LONG-PERIOD $d y$ MUTANT}

The $d y$ mutants have wing-cell sizes that are markedly smaller than normal; this led Newby et al. (1991) to hypothesize that brain cells involved in clock control might be similarly anomalous in morphology, for the subset of the $d y$ mutants whose circadian pacemakers run too slowly. We

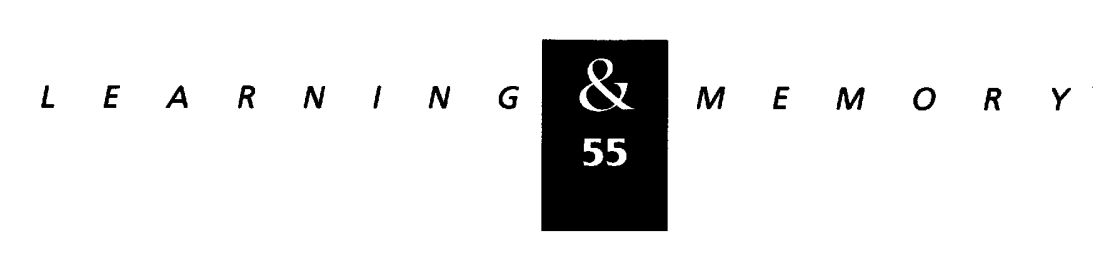


therefore examined elements of CNS anatomy in the canonical $d y^{\text {And }}$ mutant. First, a general tissue stain revealed no gross abnormalities in the morphology of ganglia in the adult head, neither in the central brain nor the four pairs of optic lobes flanking it (Fig. 2). Using these same sections, the overall volumes of wild-type and mutant brains were estimated and found to be indistinguishable (see legend to Fig. 2). Second, we scrutinized certain anatomical details associated with particular clusters of brain neurons in the brain, which comprise significant components of the fly's circadian pacemaker system (Siwicki et al. 1988; Zerr et al. 1990, Ewer et al. 1992; Frisch et al. 1994). The $\sim 25$ pairs of such neurons, distributed among 3 pairs of bilaterally symmetrical clusters, are conveniently marked by the reporter factor contained in a perlac $Z$ fusion gene; $\beta$ GAL activity or immunoreactivity stains mostly the cell bodies of these putative pacemaker neurons (e.g., Liu et al. 1988, 1991; Ewer et al. 1992; Helfrich-Förster 1995).

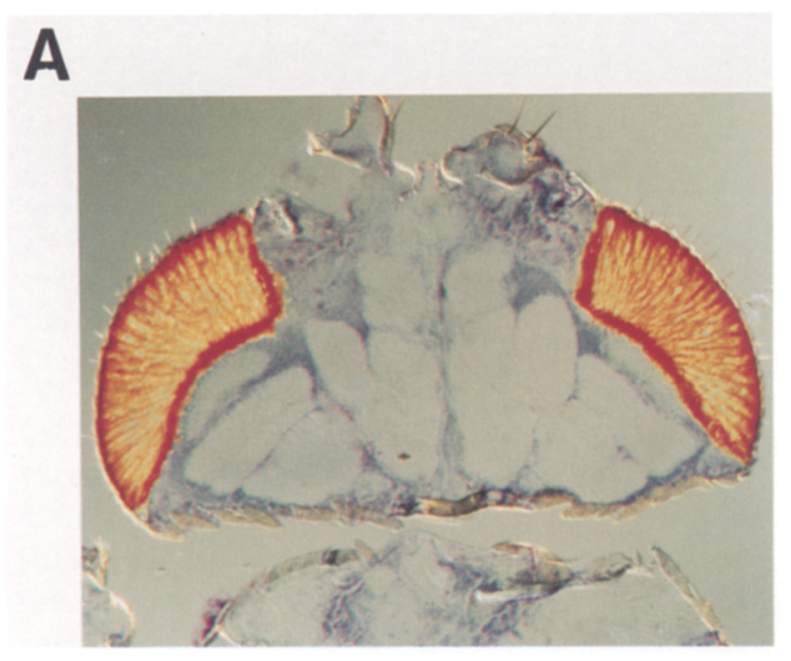

The per-expressing pacemakers also are stained fortuitously by application of an anti-ARRESTIN antibody (see Materials and Methods), which marks the cell bodies and also neuronal processes emanating from these "per-neuronal" perikarya.

The approximate numbers and intrabrain positions of per neurons in $d y^{A n d}$ individuals were scored; these assessments were augmented by measurements of the perikaryal diameters. Typically, these relatively large cell bodies-most notably in lateral-brain regions near the anterior rim of the medulla optic lobe-are $\sim 6-10 \mu \mathrm{m}$ in diameter (e.g., Ewer et al. 1992 and size estimates done in the current experiments on sections through $d y^{+}$heads). From scrutinizing serial sections prepared from five $d y^{A n d}$; per-lac $Z$ adults, no abnormalities in numbers, positions, or lateralneuronal diameters could be discerned (Fig. 3; see its legend for information on per-lacZ-mediated signals in controls). The cell-body measurements were made on 15 such neurons, with two to four

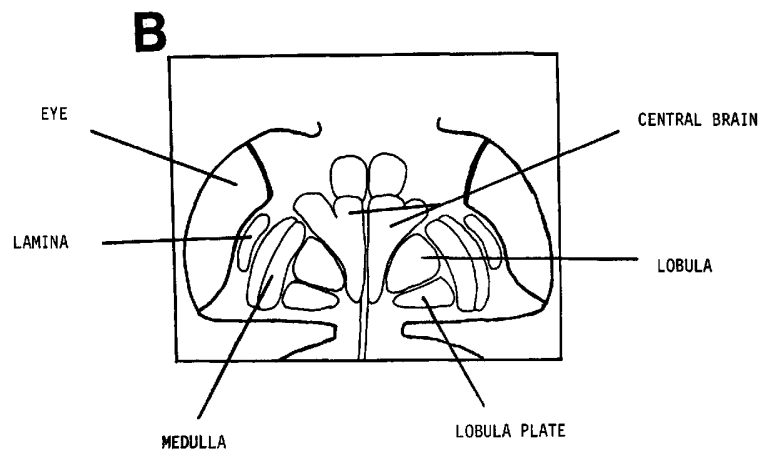

Figure 2: General brain morphology of the $d y^{\text {And }}$ mutant. (A) A horizontal section from a $d y^{\text {And }}$ male's adult head was stained with Toluidine blue. Notwithstanding the low resolution nature of this image, the overall morphology of the brain and the various ganglia and lobes within it look normal; the same was so for six additional $d y^{\text {And }}$ males sectioned and stained in this manner or with Methylene blue. (B) A drawing of a horizontal brain section from a normal adult (representing eight such controls sectioned and stained in this manner), which indicates the major substructures within the central brain and visual system. The distance between the most distal regions of the paired lamina optic lobes is $\sim 450 \mu \mathrm{m}$. Actual measurements of $d y^{A n d}$ and $d y^{+}$CNS widths were made-at this rather central position (re a series of horizontal sections), and at every fifth section, proceeding more dorsally and more ventrally from it. The average width for the entire CNS/optic lobe complex (measured from just inside the retinal layers on either side of the brain) was $406 \pm 46$ (S.E.M.) $\mu \mathrm{m}$ for wild type $(n=8)$ and $399 \pm 37 \mu \mathrm{m}$ for $d y^{A n d}(n=7)$; these values are means of means, i.e., based on measurements of seven horizontal sections per specimen (the intrafly variability, with respect to standard deviations; for such measurements were $\sim 20 \%$ of the per-fly mean values, reflecting much smaller widths in the dorsal-most sections). Average widths for the CNS proper were $169 \pm 17 \mu \mathrm{m}$ for wild type and $160 \pm 13 \mu \mathrm{m}$ for $d y^{\text {And }}$ (intrafly variability among planes of section was $\sim 30 \%$ of the means, owing to the fact that the central brain, ignoring the flanking optic lobes, is relatively spherical, giving low values in both dorsal and ventral regions).

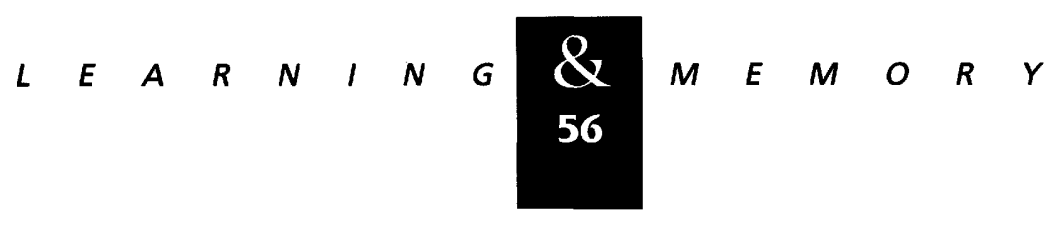



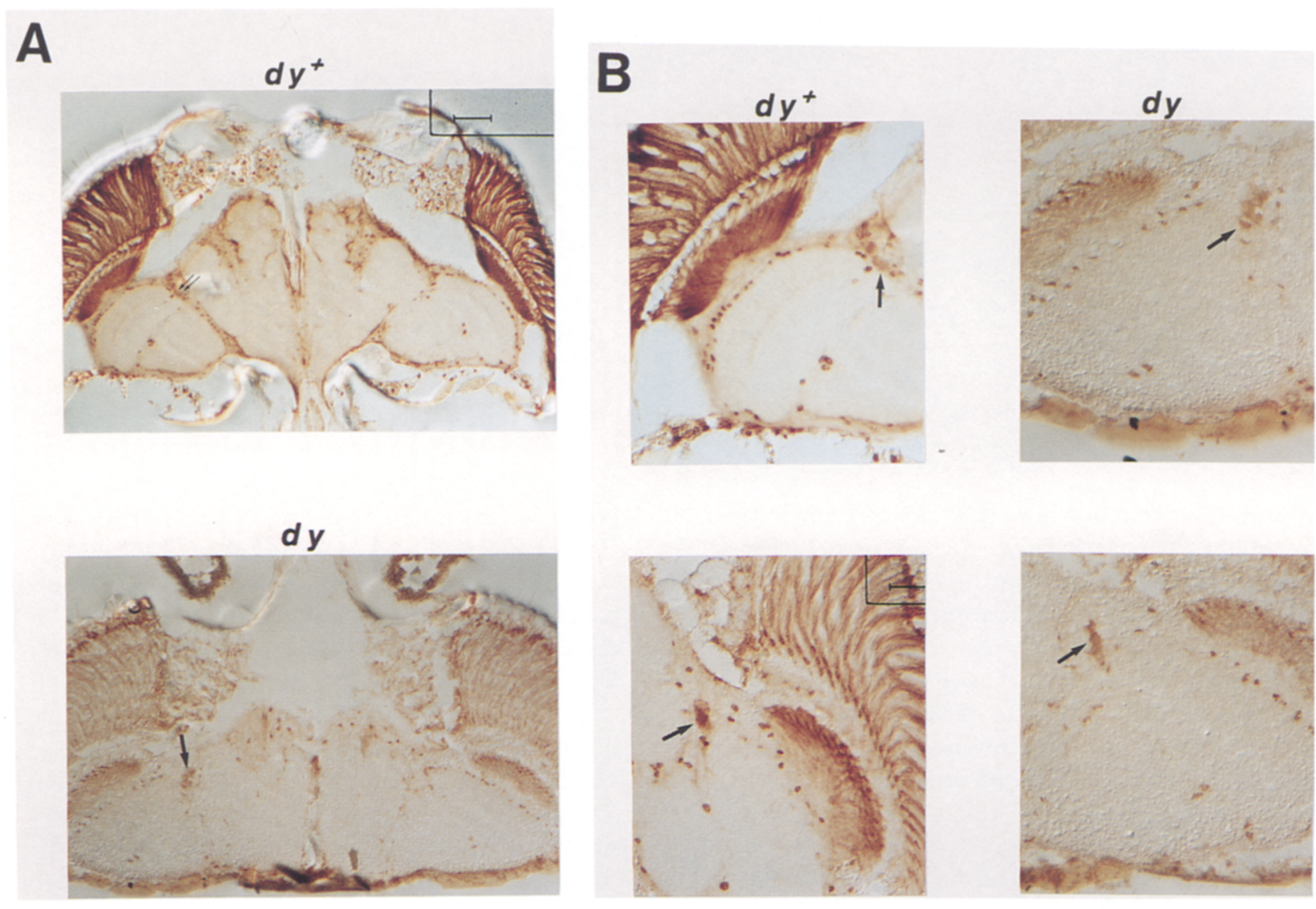

Figure 3: Overall and rhythm-related anatomy of brains. (A) A horizontal section through the head of a per-lac $Z$ fusion transgenic male, compared with one from a $d y^{\text {And }}$; per-lac $Z$ male; both were stained with anti- $\beta$-galactosidase at approximately the same level as the Toluidine-blue stain in Fig. 2. (Arrows) Lateral neurons (cf. Table 3), which are larger and fuzzier in appearance than neighboring smaller cells, the latter being glia (cf. Ewer et al. 1992). The control (dy ${ }^{+}$) fly, representing 12 adults of this genotype that were processed in this manner, carried two copies of the reporter-fusion gene, which is noticeable by its darker stain. Lateral neurons are nevertheless evident in the $d y^{\text {And }}$ fly whose section is shown here, as they were in the others of this genotype that were sectioned and stained $(n=10)$. Bar, for the $d y^{+}$section, $40 \mu \mathrm{m}$; that from the $d y$ fly is shown at $1.2 \times$ the control image. (B) Lateral neurons (cf. Table 3 ) in control per-lac $Z$ and $d y$ And; per-lacZ flies. These cells, observed in all five And flies assayed, had diameters ranging from 6 to $10 \mu \mathrm{m}$ (with respect to 15 cells sampled)_essentially identical to what was observed in contemporary controls and in previous studies (notably Ewer et al. 1992). Bar, (bottom left), $14 \mu \mathrm{m}$; the other control section directly above is shown at $0.75 \times$ this magnification. The two $d y$ images are at $1.5 \times$ that of the scale-barred magnification.

perikarya being sampled per fly, from among five separate adults of the genotype noted above; all such diameters were in the normal 6- to $10-\mu \mathrm{m}$ range. Five additional $d y^{A n d}$ adults were sectioned and stained using $\alpha$-ARR2. The antigen (a crossreacting one, as opposed to the arr2's gene product; see Materials and Methods) was distributed in CNS patterns similar to those observed after application of this antibody to head sections of wildtype adults (Fig. 4; see its legend for information on control immunoreactivity). Moreover, various per-neuronal "parameters" were determined for the $\alpha$-ARR2-stained controls and mutants (Table 3 ). These quasinumerical details-referring to cell bodies and axonal projections stained by this antibody, including those that seem to be aimed at the dorsal mushroom bodies (see Table 3 footnote; cf. Helfrich-Förster and Homberg 1993)showed no $d y^{A n d}$-induced abnormalities.

\section{Discussion}

The dy mutants of Drosophila melanogaster are anatomical ones by definition. In this regard,

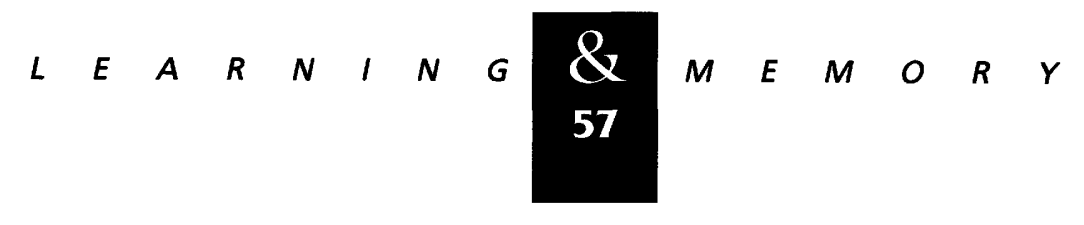



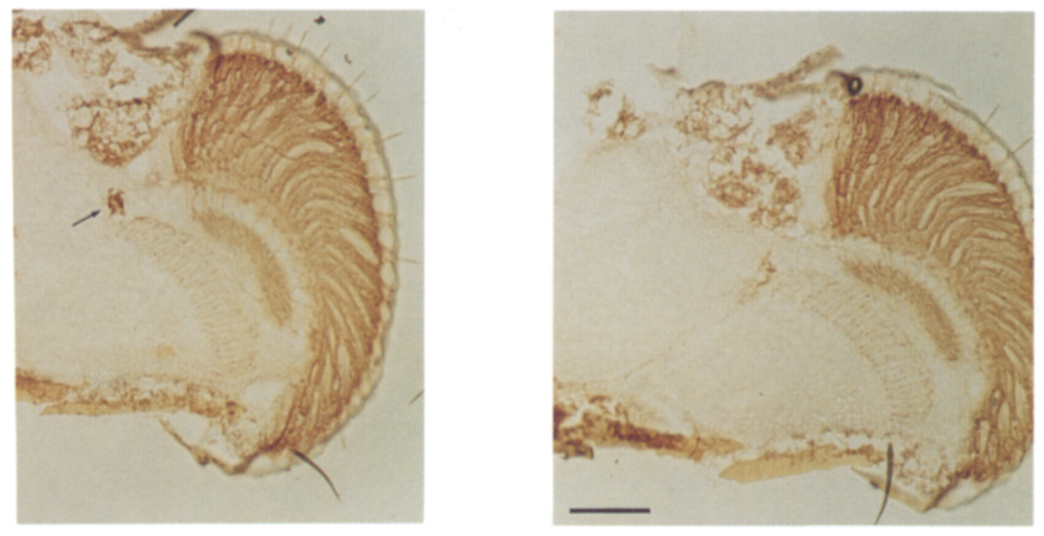

Figure 4: ARRESTIN-2-like immunoreactivity in $d y^{\text {And }}$. Two consecutive horizontal sections, at 140-150 $\mu \mathrm{m}$ from the dorsal-most part of the brain, are depicted, having been exposed to the $\alpha$-ARR2 reagent that leads to staining of bona fide ARRESTIN-2 in the eye and of cross-reacting material in the CNS, including the LN cells shown. Bar, $50 \mu \mathrm{m}$.

the number of mutants in this species with abnormalities of external appearance and internal anatomy is legion (Bate and Martinez-Arias 1993). This would be one perspective from which to surmise that the $d y^{A n d}$ mutation could produce both learning and rhythm defects, in addition to considering the more specific hypothesis about relationships that may exist between the mechanisms subserving conditioning and timing phenomena. Furthermore, such a pleiotropic effect could now be rationalized, given the observation that elements of the fly's CNS involved in learning and rhythms are, although spatially distinct, literally connected (Helfrich-Förster and Homberg 1993; HelfrichFörster 1995; cf. Davis 1993; Hall 1995).

Another less interesting possibility is that general brain damage, associated with the more pleiotropically acting $d y$ mutations ( $d y^{\text {And }}$ and the like; Table 1), would disrupt the neural substrates of both courtship-related learning and circadian rhythms in a rather gratuitous fashion, as the mutational effects "swept through" a variety of brain structures. Two further points about $d y$-related pleiotropy should be mentioned in this regard: The relevant slow-clock mutations not only "should" affect something in the head (as well as of course causing defects in the development of a thoracic structure); in addition, a genetic-mosaic analysis has shown that $d y^{\text {And }}$ does indeed cause its circadian-rhythm abnormalities through a direct head effect of the mutation, with the aberrant wing morphology being autonomous to that relatively posterior appendage (Newby and Jackson 1995). Moreover, this very mutation was shown by Kyriacou et al. (1993) to cause a lengthening of the male's courtship song-rhythm period (which normally has an $\sim 55$-sec cycle duration); that behavioral defect can be inferred to have a thoracic etiology (again, from mosaic experiments) (see Hall 1984, 1995; Hall and Kyriacou 1990).

Some of the issues just discussed are moot, because none of the $d y$ mutations was found to cause decrements in conditioned courtship behavior. We should note, however, that Jackson et al. (1983) also examined courtship suppression in the immature-male assay (cf. Gailey et al. 1982; Tompkins 1989), and there, as well as in the case of mated females, found $\boldsymbol{d y} \boldsymbol{y}^{\text {And }}$ to be conditioned subnormally. Though we did not perform tests involving training of long-period $d y$ mutants in the presence of such immature males, our results (Table 2) further weaken the notion that, in Drosophila, the control of rhythms and learning share underlying genetic factors, that is, in addition to the weakening that resulted from a reexamination of slow-clock per mutants in courtship-suppression assays (Gailey et al. 1991).

An irony here is that chronomnemonic connections recently have been reforged in the opposite genetic direction: dunce learning/memory mutations were shown to cause a mild alteration of free-running rhythm periodicity and a more substantial one relating to light-pulse pacemakerresetting dynamics (Levine et al. 1994). Moreover, DCO mutants, which show reductions of protein kinase $A$ activity and olfactory learning abilities (Skoulakis et al. 1993), are also rhythm defective (Levine et al. 1994).

The $d y$ mutants' pleiotropies, which are well established insofar as external morphology and circadian rhythms are concerned, could have been reasonably predicted to extend into other areas, just as dunce's pleiotropy goes even beyond the phenotypes noted above (for review, see Hall 1994b). Thus, $d y$ mutants might have been demonstrable as globally defective genetic variants

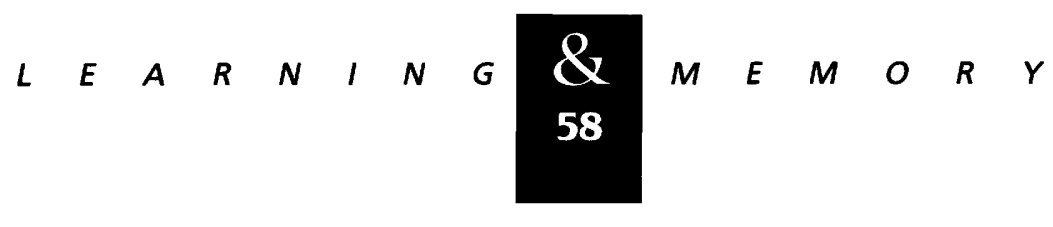


that happen to encompass learning abnormalities among their several impairments. This is a somewhat different view of the gene's hypothetically broad effects, compared with one that would focus on potentially more discrete overlaps between the fly's timing and learning "machineries". In any case, neither $d y$ nor any of the other salient rhythm mutations are as behaviorally pleiotropic as the literature on them has implied (summarized most recently by Jackson 1993). And the absence of detectable $d y$-induced brain damage (Figs. 2-4), especially within fiber pathways that putatively connect circadian-pacemaker cells to other brain regions (Table 3; cf. Helfrich-Förster and Homberg 1993), once more militates against wide-ranging pleiotropy being a hallmark of the phenotypes influenced by this gene.

What about the extent to which products of the $d y$ locus are expressed, possibly in several tissue types and at various stages of the life cycle? This will have to await more definitive findings on the nature of the transcription units present at this locus: $\boldsymbol{d y}$ 's molecular genetics has been problematical (Jackson and Newby 1993). Once the complexities in question are unraveled-and against a background of $d y$ mutants being wing only or wings and rhythms-it may turn out that the gene products are found in rather discrete locations. The results of our reexamination of the possibility that $d y$ 's effects spread beyond its clear areas of action are consistent with the idea that this gene's pleiotropy, and hence its directly revealed expression patterns, may turn out to be rather limited.

\section{Acknowledgments}

We thank the following colleagues for experimental help in these areas: Melanie J. Hamblen-Coyle for rhythm-testing; Adriana Villella and Donald A. Gailey for courtship conditioning; Brigitte Frisch for histology; Tim Tully for statistics. We appreciate comments on the manuscript from Ralph J. Greenspan. This work was supported by a grant from the National Institutes of Heath (GM-21473).

The publication costs of this article were defrayed in part by payment of page charges. This article must therefore be hereby marked "advertisement" in accordance with 18 USC section 1734 solely to indicate this fact.

\section{References}

Bate, M. and A. Martinez-Arias, ed. 1993. The Development of Drosophila melanogaster, Vols. I and II. Cold Spring Harbor Laboratory Press, Cold Spring Harbor, New York.

Baylies, M.K., T.A. Bargiello, F.R. Jackson, and M.W. Young. 1987. Changes in abundance or structure of the per gene product can alter periodicity of the Drosophila clock. Nature 326: 390-392.

Bouhouche, A. and M.K. Choulli. 1994. Étude de l'inhibition sexuelle acquise chez un mutant neurologique, no-bridge ${ }^{\kappa S 49}$, appartenant à l'espèce Drosophila melanogaster. Can. J. Zool. 72: 1376-1382.

Collins, M.F. and J.K. Hewitt. 1981. A biometrical analysis of a reported conditioned response in the courtship behaviour of male Drosophila melanogaster. Behav. Genet. 11: 595-596.

Cowan, T.M. and R.W. Siegel. 1984. Mutational and pharmacological alterations of neuronal membrane function disrupt conditioning in Drosophila. I. Neurogenet. 1: 333-344.

Davis, R.L. 1993. Mushroom bodies and Drosophila learning. Neuron 11: 1-14.

de Belle, J.S. and M. Heisenberg. 1994. Associative odor learning in Drosophila abolished by chemical ablation of mushroom bodies. Science 263: 692-695.

Dolph, P.J., R. Ranganathan, N.J. Colley, R.W. Hardy, M. Socolich, and C.S. Zuker. 1993. Arrestin function in inactivation of $G$ protein-coupled receptor rhodopsin in vivo. Science 260: 1910-1916.

Ewer, J., B. Frisch, M.J. Hamblen-Coyle, M. Rosbash, and J.C. Hall. 1992. Expression of the period clock gene within different cell types in the brain of Drosophila adults and mosaic analysis of these cells' influence on circadian behavioral rhythms. J. Neurosci. 12: 3321-3349.

Frisch, B., P.E. Hardin, M.J. Hamblen-Coyle, M. Rosbash, and J.C. Hall. 1994. A promoterless period gene mediates behavioral rhythmicity and cyclical per expression in a restricted subset of the Drosophila nervous system. Neuron 12: $555-570$.

Gailey, D.A. and R.W. Siegel. 1989. A mutant strain in Drosophila melanogaster that is defective in courtship behavioural cues. Anim. Behav. 38: 163-169.

Gailey, D.A., F.R. Jackson, and R.W. Siegel. 1982. Male courtship in Drosophila: The conditioned response to immature males and its genetic control. Genetics 102: $771-782$.

1984. Conditioning mutations in Drosophila melanogaster affect an experience-dependent behavioral modification in courting males. Genetics 106: 613-623.

Gailey, D.A., R.C. Lacaillade, and J.C. Hall. 1986. Chemosensory elements of courtship in normal and mutant, olfaction-deficient Drosophila melanogaster. Behav. Genet. 16: 375-405.

Gailey, D.A., A. Villella, and T. Tully. 1991. Reassessment of the effect of biological rhythm mutations on learning in Drosophila melanogaster. J. Comp. Physiol. A 169: 685-697.

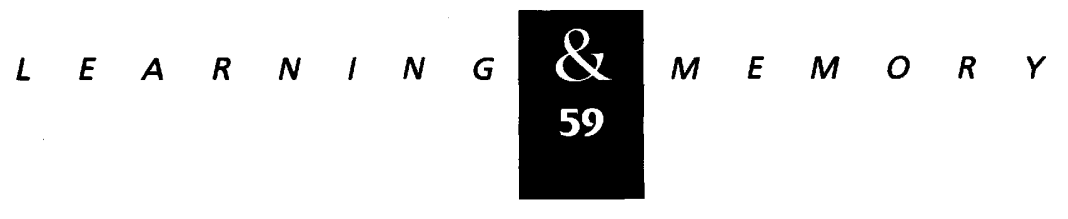


van Swinderen and Hall

Greenspan, R.J. 1995. Understanding the genetic construction of behavior. Sci. Amer. 272:(4) 88-93.

Griffith, L.C., L.M. Verselis, K.M. Aitken, C.P. Kyriacou, W. Danho, and R.J. Greenspan. 1993. Inhibition of calcium/calmodulin-dependent protein kinase in Drosophila disrupts behavioral plasticity. Neuron 10: 501-509.

Griffith, L.C., J. Wang, Y. Zhong, C.-F. Wu, and R.J. Greenspan. 1994. Calcium/calmodulin-dependent protein kinase II and potassium channel subunit Eag similarly affect plasticity in Drosophila. Proc. Nat. Acad. Sci. 91: 10044-10048.

Grossman, M. and H.W. Norton. 1980. Approximate intrinsic bias in estimates of heritability based on variance component analysis. J. Hered. 71: 295-297.

Hall, J.C. 1984. Complex brain and behavioral functions disrupted by mutations in Drosophila. Dev. Genet. 4: $355-378$.

1994a. The mating of a fly. Science 264: 1702-1714.

1994b. Pleiotropy of behavioral genes. In Flexibility and constraint in behavioral systems (ed. R.J. Greenspan and C.P. Kyriacou), pp. 15-27. Wiley, New York.

1995. Tripping along the trail to the molecular mechanisms of biological clocks. Trends Neurosci. 18: $230-240$.

Hall, J.C. and C.P. Kyriacou. 1990. Genetics of biological rhythms in Drosophila. Adv. Insect Physiol. 22: 221-298.

Hamblen, M., W.A. Zehring, C.P. Kyriakou, P. Reddy, Q. Yu, D.A. Wheeler, L.J. Zwiebel, R.J. Konopka, M. Rosbash, and J.C. Hall. 1986. Germ-line transformation involving DNA from the period locus in Drosophila melangaster: Overlapping genetic fragments that restore circadian and ultradian rhythmicity to per ${ }^{\circ}$ and per- mutants. I. Neurogenet. 3: 249-291.

Hamblen-Coyle, M.J., D.A. Wheeler, J.E. Rutila, M. Rosbash, and J.C. Hall. 1992. Behavior of period-altered circadian rhythm mutants of Drosophila in light:dark cycles (Diptera: Drosophilidae). J. Insect Behav. 5: 417-446.

Heisenberg, M. 1980. Mutants of brain structure and function: What is the significance of the mushroom bodies for behavior? In Development and neurobiology of Drosophila (ed. O. Siddiqi, P. Babu, L.M. Hall, and J.C. Hall), pp. 373-390. Plenum Press, New York.

Helfrich-Förster, C. 1995. The period clock gene is expressed in CNS neurons which also produce a neuropeptide that reveals the projections of pacemaker cells within the brain of Drosophila melanogaster. Proc. Natl. Acad. Sci.

92: 612-616.

Helfrich-Förster, C. and U. Homberg. 1993.

Pigment-dispersing hormone-immunoreactive neurons in the nervous system of wild-type Drosophila melanogaster and of several mutants with altered circadian rhythmicity. J. Comp. Neurol. 337: 177-190.

Hofbauer, A. and E. Buchner. 1989. Does Drosophila have seven eyes? Naturwissenschaften 76: 335-336.

Huang, J.Z., I. Edery, and M. Rosbash. 1993. PAS is a dimerization domain common to Drosophila Period and several transcription factors. Nature 364: 259-262.

Jackson, F.R. 1993. Circadian rhythm mutants of Drosophila. In Molecular genetics of biological rhythms (ed. M.W. Young), pp. 91-121. Marcel Dekker, New York.

Jackson, F.R. and L.M. Newby. 1993. Products of the Drosophila miniature-dusky gene complex function in circadian rhythmicity and wing development. Comp. Biochem. Physiol. 104A: 749-756.

Jackson, F.R., D.A. Gailey, and R.W. Siegel. 1983. Biological rhythm mutations affect an experience-dependent modification of male courtship behavior in Drosophila melanogaster. J. Comp. Physiol. A 151: 545-552.

Jenkins, J. and K. Dallenbach. 1924. Oblivescence during sleep and waking. Amer. I. Psychol. 35: 605-612.

Konopka, R.J. 1987. Genetics of biological rhythms in Drosophila. Annu. Rev. Cenet. 21: 227-236.

Konopka, R.J., R.F. Smith, and D. Orr. 1991.

Characterization of Andante, a new Drosophila clock mutant, and its interactions with other clock mutants. J. Neurogenet. 7: 103-114.

Kyriacou, C.P., M.L. Greenacre, J.R. Thackeray, and J.C. Hall. 1993. Genetic and molecular analysis of song rhythms in Drosophila. In Molecular genetics of biological rhythms (ed. M.W. Young), pp. 171-193. Marcel Dekker, New York.

Levine, J.D., C.I. Casey, D.D. Kalderon, and F.R. Jackson. 1994. Altered circadian pacemaker functions and cyclic AMP rhythms in the Drosophila learning mutant dunce. Neuron 13: $967-974$.

Liu, X., L. Lorenz, Q. Yu, J.C. Hall, and M. Rosbash. 1988. Spatial and temporal expression of the period gene in Drosophila melanogaster. Genes \& Dev. 2: 228-238.

Liu, X., Q. Yu, Z. Huang, L.J. Zwiebel, J.C. Hall, and M. Rosbash. 1991 The strength and periodicity of $\mathrm{D}$. melanogaster circadian rhythms are differentially affected by alterations in period gene expression. Neuron 6: 753-766.

Newby, L.M. and F.R. Jackson. 1995. Developmental and genetic mosaic analysis of Drosophila $m$ - $d y$ mutants: Tissue foci behavioral and morphogenetic defects. Dev. Genet. 16: 85-93.

Newby, L.M., L. White, S.M. DiBartolomeis, B.J. Walker,

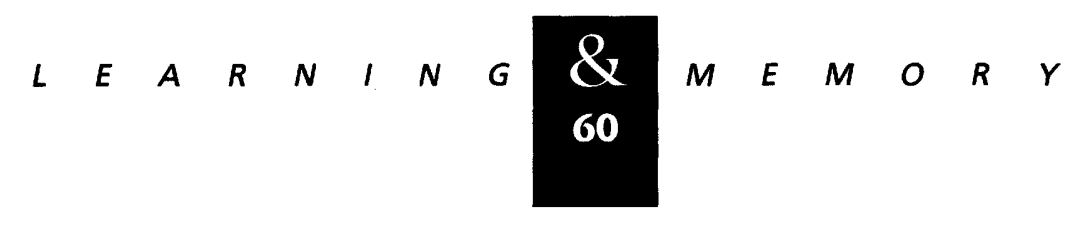


H.B. Dowse, J.M. Ringo, N. Khuda, and F.R. Jackson. 1991 Mutational analysis of the Drosophila miniature-dusky ( $m-d y)$ locus: Effects on cell size and circadian rhythms. Genetics 128: $571-582$.

O'Dell, K.M.C. 1994. The inactive mutation leads to abnormal experience-dependent courtship modification in male Drosophila melanogaster. Behav. Genet. 24: 381-388.

Siegel, R.W., J.C. Hall, D.A. Gailey, and C.P. Kyriacou. 1984. Genetic elements of courtship in Drosophila: Mosaics and learning mutants. Behav. Genet. 14: 383-410.

Siwicki, K.K., C. Eastman, G. Petersen, M. Rosbash, and J.C. Hall. 1988. Antibodies to the period gene product of Drosophila reveal diverse tissue distribution and rhythmic changes in the visual system. Neuron 1: 141-150.

Skoulakis, E.M., D. Kalderon, and R.L. Davis. 1993. Preferential expression in mushroom bodies of the catalytic subunit of protein kinase $A$ and its role in learning and memory. Neuron 11: 197-208.

Smith, C. 1985. Sleep states and learning: A review of the animal literature. Neurosci. Behav. Rev. 9: 157-168.

Sokal, R.R. and F.J. Rohlf. 1981. Biometry, 2nd ed. p. 241, pp. 427-428, W.H. Freeman \& Co., New York.

Stone, W.S., H.J. Altman, R.F. Berman, D.F. Caldwell, and M.M. Kilbey. 1989. Association of sleep parameters and memory in intact old rats and young rats with lesions in the nucleus basalis magnocellularis. Behav. Neurosci.

103: 755-764.

Tempel, B.L., M.S. Livingstone, and W.G. Quinn. 1984. Mutations in the dopa decarboxylase gene affect learning in Drosophila. Proc. Natl. Acad. Sci. 81: 3577-3581.

Tompkins, L. 1984. Genetic analysis of sex appeal in Drosophila melanogaster. Behav. Genet. 14: 453-482.

1989. Homosexual courtship in Drosophila. In Perspectives in neural systems and behavior (ed. T.J. Carew and D. Kelley), pp. 229-248. Alan R. Liss, New York.

Tully, T. and J. Hirsch. 1982. Behaviour-genetic analysis of Phormia regina II. Detection of a single, major-gene effect from behavioural variation for central excitatory state (CES) using hybrid crosses. Anim. Behav. 30: 1193-1202.

Wheeler, D.A., M.J. Hamblen-Coyle, M.S. Dushay, and J.C. Hall. 1993. Behavior in light-dark cycles of Drosophila mutants that are blind, arrhythmic, or both. J. Biol. Rhythms 8: 67-94.

Zawistowski, S. 1988. A replication demonstrating reduced courtship of Drosophila melanogaster by associative learning. J. Comp. Psychol. 102: 174-176.

Zawistowski, S. and R.C. Richmond. 1985. Experience mediated courtship reduction for males by male Drosophila melanogaster. Behav. Genet. 15: 561-569.
1988. Experience-dependent reduction in courtship of Drosophila melanogaster in large and small chambers. $J$. Comp. Psychol. 101: 90-93.

Zerr, D.M., J.C. Hall, M. Rosbash, and K.K. Siwicki. 1990. Circadian fluctuations of the period protein immunoreactivity in the CNS and the visual system of Drosophila. J. Neurosci. 10: $2749-2762$.

Received April 4, 1995; accepted in revised form May 31, 1995. 


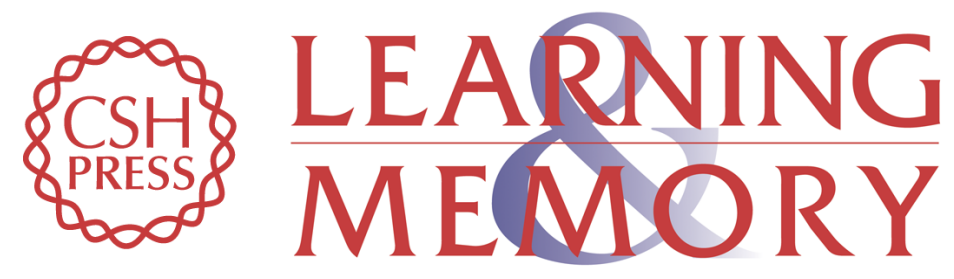

\section{Analysis of conditioned courtship in dusky-Andante rhythm mutants of Drosophila.}

$\mathrm{B}$ van Swinderen and J C Hall

Learn. Mem. 1995, 2:

Access the most recent version at doi:10.1101//m.2.2.49

References This article cites 52 articles, 12 of which can be accessed free at: http://learnmem.cshlp.org/content/2/2/49.full.html\#ref-list-1

License

Email Alerting Receive free email alerts when new articles cite this article - sign up in the box at the Service top right corner of the article or click here. 